OPEN ACCESS

Edited by:

Niccolo Petrucciani,

Sapienza University of Rome, Italy

Reviewed by:

Aya Kuchiba,

National Cancer Centre, Japan

Tadahiko Masaki,

Kyorin University, Japan

Peter Gibbs,

Walter and Eliza Hall Institute of

Medical Research, Australia

*Correspondence:

Xu Guan

drguanxu@163.com

Zheng Jiang

812818858@qq.com

Xi-shan Wang

wxshan_1208@126.com

tThese authors have contributed equally to this work

Specialty section:

This article was submitted to Gastrointestinal Cancers,

a section of the journal

Frontiers in Oncology

Received: 17 March 2020

Accepted: 18 May 2020

Published: 19 June 2020

Citation:

Ma C, Guan X, Wei R, Wang S, Quan

J, Zhao Z, Chen H, Liu Z, Jiang Z and

Wang $X$ (2020) The Distinction of

Clinicopathological Characteristics,

Treatment Strategy and Outcome in Colorectal Cancer Patients With

Synchronous vs. Metachronous Bone

Metastasis. Front. Oncol. 10:974.

doi: 10.3389/fonc.2020.00974

\section{The Distinction of Clinicopathological Characteristics, Treatment Strategy and Outcome in Colorectal Cancer Patients With Synchronous vs. Metachronous Bone Metastasis}

\author{
Chen-xi Ma ${ }^{1}$, Xu Guan ${ }^{1 * t}$, Ran Wei ${ }^{1}$, Song Wang ${ }^{2}$, Ji-chuan Quan ${ }^{1}$, Zhi-xun Zhao', \\ Hai-peng Chen ${ }^{1}$, Zheng Liu ${ }^{1}$, Zheng Jiang ${ }^{1 * t}$ and Xi-shan Wang ${ }^{1 * t}$

\footnotetext{
${ }^{1}$ Department of Colorectal Surgery, National Cancer Center/National Clinical Research Center for Cancer/Cancer Hospital, Chinese Academy of Medical Sciences and Peking Union Medical College, Bejing, China, ${ }^{2}$ Department of Colorectal Surgery, The Second Affiliated Hospital of Harbin Medical University, Harbin, China
}

Background: The impact of the timing of bone metastasis (BM) diagnosis on colorectal cancer (CRC) patients is unclear. Our study aimed to explore the differences in clinicopathological characteristics, treatments and prognosis between synchronous BM (SBM) and metachronous BM (MBM) from CRC.

Methods: We retrospectively investigated clinical data of CRC patients with SBM or MBM from 2008 to 2017 at Chinese National Cancer Center. Cancer specific survival (CSS) after BM diagnosis was estimated using the Kaplan-Meier method. The multivariable COX regression model identified the prognostic factors of CSS.

Results: Finally, 63 CRC patients with SBM and 138 CRC patients with MBM were identified. Compared to SBM from CRC, MBM significantly was more involving multiple bone lesions (63.0 vs. $7.9 \% ; p<0.001$ ), and more frequently originated from rectal cancer (60.9 vs. $41.3 \% ; p=0.033$ ). The therapeutic strategies in SBM and MBM group were contrasted including systemic treatment, bisphosphonates, radiotherapy and metastasectomy for BM. $85.5 \%$ of patients in MBM group and $25.4 \%$ of patients in SBM group underwent primary tumor resection at initial diagnosis $(p<0.001)$. The median CSS was 11 months in both SBM and MBM group ( $p=0.556)$, yet MBM patients developed from CRC in early AJCC stage presented obviously longer survival than those from advanced stage. Furthermore, patients could have improved CSS from primary tumor resection while there might be no survival benefit from targeted therapy in both SBM and MBM groups. Bisphosphonates was associated with a better CSS for patients with SBM, while radiotherapy for BM was related to a better CSS for patients with MBM.

Conclusion: The CRC patients in SBM and MBM group represented different clinicopathological characteristics and treatment modalities, which affected the prognosis in different ways. Distinct consideration for CRC patients with SBM and MBM in clinical decision making is required.

Keywords: colorectal cancer, bone metastasis, synchronous, metachronous, cancer specific survival, prognostic factor 


\section{INTRODUCTION}

Colorectal cancer (CRC) with distant metastasis is one of the main causes of death. About 20\% of CRC patients are diagnosed with distant metastasis at initial diagnosis and 50$60 \%$ will eventually have metastases $(1,2)$. The CRC commonly metastasizes to liver, followed by lung, yet seldom to bone (3). Population-based studies have reported the incidence of BM is $3.0-10.4 \%$ in CRC patients (4-6), but previous autopsy findings have suggested incidence of up to $23.7 \%$ (7). The prognosis after BM detection is generally poor due to the advanced stage and the difficulty in treatment, with 5 -year survival rate $<5 \%$ (8). Median overall survival of CRC patients after BM diagnosis ranges from 5 to 22 months according to most researches (4, 9), with diverse factors affecting their prognosis such as some clinicopathological characteristics and provision of treatment. However, there is a lack of standard treatment guideline for BM from CRC at present. The possible therapies for BM include systemic therapy, local therapy and supportive treatment, with purpose to prevent skeletal-related events (SREs) like sever bone pain, hypercalcemia, spinal cord compression and pathological fracture and improve the survival of patients.

Synchronous BM (SBM) in CRC patients is relatively rare while most BMs occur metachronously after a length of followup time or during palliative treatment for other metastases. Generally, the patients with metachronous BM (MBM) have received systematic clinical intervention before the osseous lesion development, whereas those with SBM are mostly naive. Therefore, SBM and MBM from CRC may represent distinct clinicopathological characteristics, therapeutic sensitivity and outcomes, which require different treatment strategies. Many reports are controversial on the outcomes of synchronous and metachronous metastases from CRC, and most of which agree about the more aggressive clinical and pathological features of synchronous metastases (10-14). However, few studies in specifically exploring the differences between SBM and MBM from CRC have been reported.

Thus, the aims of our study were to (1) compare the clinicopathological characteristics of SBM and MBM from CRC; (2) compare the treatment modalities for SBM and MBM from CRC; (3) explore outcomes and prognostic factors of CRC patients with SBM and MBM, especially the impact of various treatment modalities on their prognosis, which would be helpful in modifying clinical management.

\section{MATERIALS AND METHODS}

\section{Data Resources and Study Population}

CRC patients who were diagnosed with BM between January 2008 and December 2017 at Chinese National Cancer Center, were retrospectively identified. The primary CRC lesion was confirmed by histopathological examination. The American Joint Committee on Cancer (AJCC) TNM stage and BM were identified by histopathological or imaging examinations such as standard X-rays, whole-body bone scans, computed tomography (CT), magnetic resonance imaging (MRI) and positron emission tomography-computed tomography (PET-CT). SBM refers to $\mathrm{BM}$ found within 3 months after the diagnosis of CRC, while $\mathrm{MBM}$ refers to $\mathrm{BM}$ found more than 3 months after the diagnosis of $\operatorname{CRC}(15,16)$. For the number of BM, two adjacent vertebral metastases were classified into the solitary bone involvement, while non-consecutive metastases or more than 2 consecutive vertebral metastases were classified as multiple bone involvement. The time of follow-up was calculated from the BM diagnosis to death or January 2020. The cancer specific survival (CSS) was defined as the time from the BM diagnosis until cancer-associated death or the end of follow up. This study was approved by the Ethics Committee of Cancer Hospital, Chinese Academy of Medical Sciences.

\section{Prognostic Factors}

Clinicopathological data and treatment methods were collected from medical records or via telephone follow-ups. Common variables were analyzed including age, gender, basic disease, primary tumor location, pathological type of tumor, tumor grade, carcinoembryonic antigen (CEA) levels at BM diagnosis, carbohydrate antigen199 (CA199) levels at BM diagnosis, alkaline phosphatase (ALP) levels at BM diagnosis, bone involvement, Karnofsky performance scores (KPS) at $\mathrm{BM}$ diagnosis, extra-osseous metastases, primary tumor resection, systemic treatment for BM, bisphosphonates for BM, radiotherapy for BM and operation for BM. Besides, AJCC TNM stage at initial diagnosis and time until BM were additionally evaluated for MBM. The basic disease was defined as other long-term or chronic coexisting diseases the BM patients from CRC suffered from, which affected basic metabolism or immune function of patients, mainly including hypertension, diabetes, heart disease, hepatitis, tuberculosis, autoimmune diseases, etc.

\section{Statistical Analysis}

The comparison of clinicopathological characteristics and treatments between patients with SBM and MBM was done using $\chi 2$-test or Fisher's exact test where appropriate. The CSS was assessed with Kaplan-Meier method, with the log-rank tests used to compare subgroups. In order to reduce the selection bias, variables with $p<0.10$ by univariate Kaplan-Meier analysis were selected first, then a forward stepwise selection was performed using the selected variables in multivariable COX regression analysis. The independent prognostic factor was defined as the variable with $p<0.05$ by COX regression. Hazard ratio (HR) and corresponding 95\% confidence interval (CI) were also calculated by multivariable COX analysis. All statistical analyses were performed with SPSS version 25.0 for Mac. It is considered as statistically significant when $p<0.05$.

\section{RESULTS}

Finally, in total of 201 patients diagnosed with BM from CRC entered in our final analysis after excluding 31 cases who were not eligible (Figure 1). 31.3\% of patients (63/201) were identified with SBM at initial diagnosis while additional $68.7 \%$ of patients (138/201) developed MBM after diagnosis of CRC. 


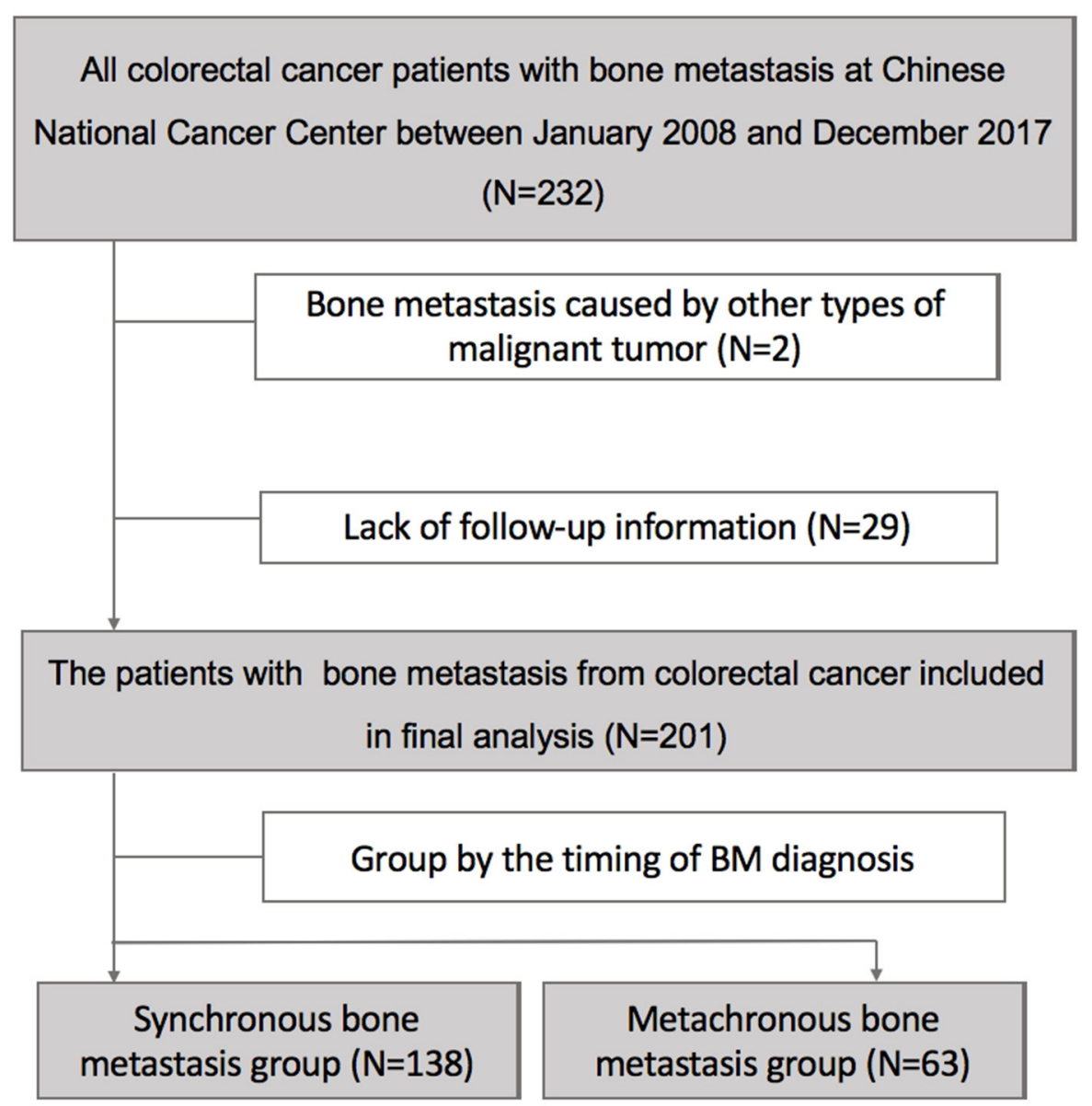

FIGURE 1 | The analytical cohort and exclusion criteria.

\section{Patients Characteristics}

Table 1 represented the clinicopathological characteristics of CRC patients with SBM and MBM. The median age of patients was 58 years (range 33-84) in SBM group and 59 years (range 19-79) in MBM group, respectively. CRC patients with SBM and MBM were similar with respect to their age at BM diagnosis $(p=0.974)$, gender $(p=0.459)$ and basic disease $(p=0.628)$. The rectal cancer was more common in MBM group $(60.9 \%)$ than SBM group (41.3\%), with statistical significance $(p=0.033)$. Patients with MBM were diagnosed more often with lower tumor grade (63.8 vs. $46.0 \% ; p=0.048$ ) compared to those with SBM. Performances in CAE levels $(p=0.511)$, CA199 levels $(p=$ $0.619)$, ALP levels $(p=0.827)$ and KPS $(p=0.631)$ at BM diagnosis between two groups were, respectively similar.

\section{Patterns of BM and Extra-Osseous Metastasis}

Patients with MBM (63.0\%) were significantly more involving multiple bone lesions compared to those with SBM $(7.9 \%$; $p<$ 0.001). Spine (65.1 vs. $73.2 \%$ ) was the leading site of BM in SBM and MBM group, followed by pelvis ( 57.1 vs. $62.0 \%$ ), long bones (34.9 vs. $22.6 \%)$ and ribs (30.2 vs. $21.9 \%)$.
There were $88.9 \%$ of patients (56/63) in SBM group and $89.1 \%$ of patients $(123 / 138)$ in MBM group having extra-osseous metastases, respectively, with no significant difference $(p=$ 0.959). The common extra-osseous sites were liver (61.9\%), distant lymph nodes (54.0\%) and lung (39.7\%) in SBM patients. While lung (57.7\%) was the most common extra-osseous metastatic site in MBM patients, followed by liver (45.7\%) and lymph nodes (40.9\%).

\section{Treatments}

There were $85.5 \%$ of MBM patients (118/138) receiving primary tumor resection, which had been all performed at initial diagnosis. The proportions of primary tumor resection in MBM patients with AJCC stage I, II, III and IV were $100.0 \%(6 / 6)$, $100.0 \%$ (14/14), 97.3\% (71/73), and 58.5\% (24/41), respectively. Of the four patients with unknown AJCC TNM stage, three received this operation. In SBM group, only $25.4 \%$ of patients $(16 / 63)$ underwent primary tumor resection because of the advanced stage.

All patients received palliative chemotherapy after BM diagnosis. There were $37.9 \%$ of cases in SBM group (25/63) and $39.1 \%$ of cases in MBM group (54/138; $p=0.941$ ) receiving 
TABLE 1 | The comparison of clinicopathological characteristics in CRC patients with SBM and MBM.

\begin{tabular}{|c|c|c|c|c|c|}
\hline \multirow[t]{2}{*}{ Variable } & \multicolumn{2}{|c|}{ Synchronous } & \multicolumn{2}{|c|}{ Metachronous } & \multirow[t]{2}{*}{$p$-value } \\
\hline & $N=63$ & $\%$ & $N=138$ & $\%$ & \\
\hline Age at BM diagnosis, years & & & & & 0.974 \\
\hline$<60$ & 35 & 55.6 & 77 & 55.8 & \\
\hline$\geq 60$ & 28 & 44.4 & 61 & 44.2 & \\
\hline Gender & & & & & 0.459 \\
\hline Female & 23 & 36.5 & 58 & 42.0 & \\
\hline Male & 40 & 63.5 & 80 & 58.0 & \\
\hline Basic disease & & & & & 0.628 \\
\hline No & 37 & 58.7 & 86 & 62.3 & \\
\hline Yes & 26 & 41.3 & 52 & 37.7 & \\
\hline Primary tumor location & & & & & 0.033 \\
\hline Rectum & 26 & 41.3 & 84 & 60.9 & \\
\hline Left hemicolon & 17 & 27.0 & 23 & 16.6 & \\
\hline Right hemicolon & 20 & 31.7 & 31 & 22.5 & \\
\hline Pathological type of tumor & & & & & $0.054^{\star}$ \\
\hline Adenocarcinoma & 56 & 88.9 & 128 & 92.8 & \\
\hline Signet-ring cell carcinoma & 4 & 6.3 & 10 & 7.2 & \\
\hline Others & 3 & 4.8 & 0 & 0.0 & \\
\hline Tumor grade & & & & & $0.048^{*}$ \\
\hline Grade I, II & 29 & 46.0 & 88 & 63.8 & \\
\hline Grade III, IV & 19 & 30.2 & 31 & 22.4 & \\
\hline UK & 15 & 23.8 & 19 & 13.8 & \\
\hline AJCC TNM stage at initial diagnosis & & & & & $<0.001^{*}$ \\
\hline I & 0 & 0.0 & 6 & 4.3 & \\
\hline ॥ & 0 & 0.0 & 14 & 10.2 & \\
\hline III & 0 & 0.0 & 73 & 52.9 & \\
\hline IV & 63 & 100.0 & 41 & 29.7 & \\
\hline UK & 0 & 0.0 & 4 & 2.9 & \\
\hline CEA levels at BM diagnosis & & & & & 0.511 \\
\hline Negative & 16 & 25.4 & 28 & 20.3 & \\
\hline Positive & 41 & 65.1 & 90 & 65.2 & \\
\hline UK & 6 & 9.5 & 20 & 14.5 & \\
\hline CA199 levels at BM diagnosis & & & & & 0.619 \\
\hline Negative & 27 & 42.9 & 50 & 36.2 & \\
\hline Positive & 29 & 46.0 & 68 & 49.3 & \\
\hline UK & 7 & 11.1 & 20 & 14.5 & \\
\hline ALP levels at BM diagnosis & & & & & 0.827 \\
\hline Negative & 47 & 74.6 & 102 & 73.9 & \\
\hline Positive & 14 & 22.2 & 29 & 21.0 & \\
\hline UK & 2 & 3.2 & 7 & 5.1 & \\
\hline Bone involvement & & & & & $<0.001$ \\
\hline Solitary & 58 & 92.1 & 51 & 37.0 & \\
\hline Multiple & 5 & 7.9 & 87 & 63.0 & \\
\hline KPS at BM diagnosis & & & & & 0.631 \\
\hline$\geq 80$ & 49 & 77.8 & 103 & 74.6 & \\
\hline$<80$ & 14 & 22.2 & 35 & 25.4 & \\
\hline Extra-osseous metastases & & & & & 0.959 \\
\hline No & 7 & 11.1 & 15 & 10.9 & \\
\hline Yes & 56 & 88.9 & 123 & 89.1 & \\
\hline
\end{tabular}

(Continued)
TABLE 1 | Continued

Variable

Synchronous Metachronous $p$-value

$N=63 \quad \% \quad N=138 \%$

Time until BM

3 months-1 year $\quad$ - $\quad$ - $\quad 45 \quad 32.6$

$1-3$ years $\quad$ - $\quad$ - $\quad 6950.0$

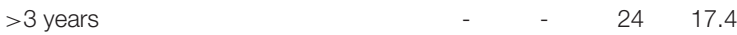

No marks indicated the $p$-value was calculated by Chi-square test and an asterisk (*) indicated the $p$-value was calculated by Fisher's test. CRC, colorectal cancer; SBM, synchronous bone metastasis: MBM, metachronous bone metastasis; $N$, number UK, unknown; CEA, carcinoembryonic antigen; CA199, carbohydrate antigen199; ALP alkaline phosphatase.

TABLE 2 | The comparison of treatment strategies in CRC patients with SBM and MBM.

\begin{tabular}{|c|c|c|c|c|c|}
\hline \multirow[t]{2}{*}{ Variable } & \multicolumn{2}{|c|}{ Synchronous } & \multicolumn{2}{|c|}{ Metachronous } & \multirow[t]{2}{*}{$p$-value } \\
\hline & $N=63$ & $\%$ & $N=137$ & $\%$ & \\
\hline Primary tumor resection & & & & & $<0.001$ \\
\hline No & 47 & 74.6 & 20 & 14.5 & \\
\hline Yes & 16 & 25.4 & 118 & 85.5 & \\
\hline \multicolumn{3}{|c|}{ Systemic treatment after BM diagnosis } & & & 0.941 \\
\hline Chemotherapy alone & 38 & 60.3 & 84 & 60.9 & \\
\hline Chemotherapy plus targeted therapy & 25 & 39.7 & 54 & 39.1 & \\
\hline Bisphosphonates for BM & & & & & 0.548 \\
\hline No & 38 & 60.3 & 77 & 55.8 & \\
\hline Yes & 25 & 39.7 & 61 & 44.2 & \\
\hline Radiotherapy for BM & & & & & 0.192 \\
\hline No & 49 & 77.8 & 95 & 68.8 & \\
\hline Yes & 14 & 22.2 & 43 & 31.2 & \\
\hline Metastasectomy for BM & & & & & $0.553^{*}$ \\
\hline No & 63 & 100.0 & 135 & 97.8 & \\
\hline Yes & 0 & 0.0 & 3 & 2.2 & \\
\hline
\end{tabular}

No marks indicated the p-value was calculated by Chi-square test and an asterisk (*) indicated the p-value was calculated by Fisher's test. CRC, colorectal cancer; SBM, synchronous bone metastasis; MBM, metachronous bone metastasis; N, number.

additional targeted therapy, respectively. The proportions of patients who received bisphosphonates treatment (60.3 vs. $55.8 \%$; $p=0.548)$ or radiotherapy ( 77.8 vs. $68.8 \% ; p=0.192)$ were, respectively, similar between two groups. Only $2.2 \%$ of patients with MBM (3/138) underwent operative treatment for BM due to spinal cord compression while no patient with SBM received metastasectomy for $\mathrm{BM}(p=0.541)$. The details were shown in Table 2.

\section{Survival}

In total of 195 CRC patients (97.0\%) died because of cancer during a median follow-up time of 11 (range 1-198) months, with 61 cases in SBM group and 134 cases in MBM group. And only one patient with MBM died due to other disease. Median CSS was both 11 months for patients with SBM and 
MBM. The median interval time from CRC diagnosis to MBM was 18.5 months. Figure 2 displayed the Kaplan-Meier curves of SBM and MBM group according to different situations. The overall CSS of patients with SBM and MBM was similar, with no significant difference ( $p=0.556$; Figure $2 \mathrm{~A}$ ). The median CSS in MBM patients with AJCC stage I, II, III and IV at initial CRC diagnosis was $28,21,10$, and 8 months, respectively, which also showed large differences compared to SBM patients $(p=0.003$; Figure 2B). In addition, patients diagnosed with $\mathrm{MBM}>3$ years after CRC diagnosis had a similar CSS with SBM patients ( $p=$ 0.093; Figure 2C).

To elucidate the outcomes with various treatments in two groups, the Kaplan-Meier curves for SBM and MBM patients were, respectively, represented in Figure 3. Patients who had underwent primary tumor resection at initial diagnosis in SBM or MBM group (Figure 3A) both had a better survival. The CSS was no significantly different between patients with and without targeted therapy in both two groups (Figure 3B). Bisphosphonates therapy was related to a better CSS in synchronous group (Figure 3C) while radiotherapy for BM (Figure 3D) was related to a better CSS in MBM group. Because only 3 patients took the osseous metastasectomy, the relationship between operation for BM and CSS was unclear.

\section{Prognostic Factors}

Table 3 showed the $p$-values obtained by univariate KaplanMeier analysis in SBM and MBM group, respectively. And the variables with $p<0.10$ were selected to be further analyzed. The independent prognostic factors $(p<0.05)$ were finally identified by multivariable COX regression analysis. We found multiple bone involvement (HR: 4.38; 95\%CI: 1.61-11.92; $p=0.002$ ), KPS scores $<80$ (HR: 2.74; 95\%CI: $1.45-5.20 ; p=0.004$ ), primary tumor resection (HR: $0.48 ; 95 \% \mathrm{CI}: 0.24-0.92 ; p=0.028)$ and bisphosphonates (HR: 0.23 ; 95\%CI: $0.12-0.43 ; p<0.001$ ) were independent prognostic factors for SBM patients (Figure 4A). While positive CA199 levels (HR: 1.92; 95\%CI: 1.30-2.83; $p=$ 0.001 ), primary tumor resection (HR: 0.50 ; $95 \%$ CI: $0.30-0.85$; $p=0.010$ ) and radiotherapy (HR: 0.53 ; 95\%CI: $0.35-0.80$; $p=0.002)$ were independent prognostic factors for MBM patients (Figure 4B).

\section{DISCUSSION}

To our knowledge, this study is the first to retrospectively analyze the SBM and MBM together from CRC patients. The MBMs were more common, with incidence nearly twice higher than SBMs. The clinicopathological characteristics differed between two groups. The most striking finding was that in total of $63.0 \%$ of patients in MBM group had BMs to multiple sites, far more than those (7.9\%) in SBM group. That might be because liver metastasis or lung metastasis from CRC in MBM group would have enough time to spread to skeletal systems by systemic circulation or directly invade chest bones such as sternum, rib and clavicle. We found there were similar therapeutic strategies between two groups, except that more MBM patients received the resection of primary tumor at initial diagnosis.

Colloca et al. (14) identified 425 CRC patients with distant metastases, discovering that the survival after metastasis diagnosis was shorter in synchronous group (18.5 vs. 62.8 months, $p<0.001$ ). Majority of reports consider synchronous metastases from CRC to be more aggressive than metachronous despite there is a controversy (10-14). In our study, the prognosis of BM was very poor, yet there was no significant difference in CSS between two groups (Figure 2A). Several reasons might explain the similar outcomes. First, a significant percentage of patients with MBM had been treated with prior chemotherapy before BM diagnosis, while patients with SBM obviously were not and they were more chemo-naive chemo-sensitive $(13,14)$. Second, the multiple bone involvement was related to worse prognosis, which was more common in MBM group. Another possibility was that BMs was so aggressive that the timing of BM diagnosis had little impact on the outcome. In addition, patients with different time intervals to MBM diagnosis had similar CSS with SBM patients as Figure 2C represented. However, it was

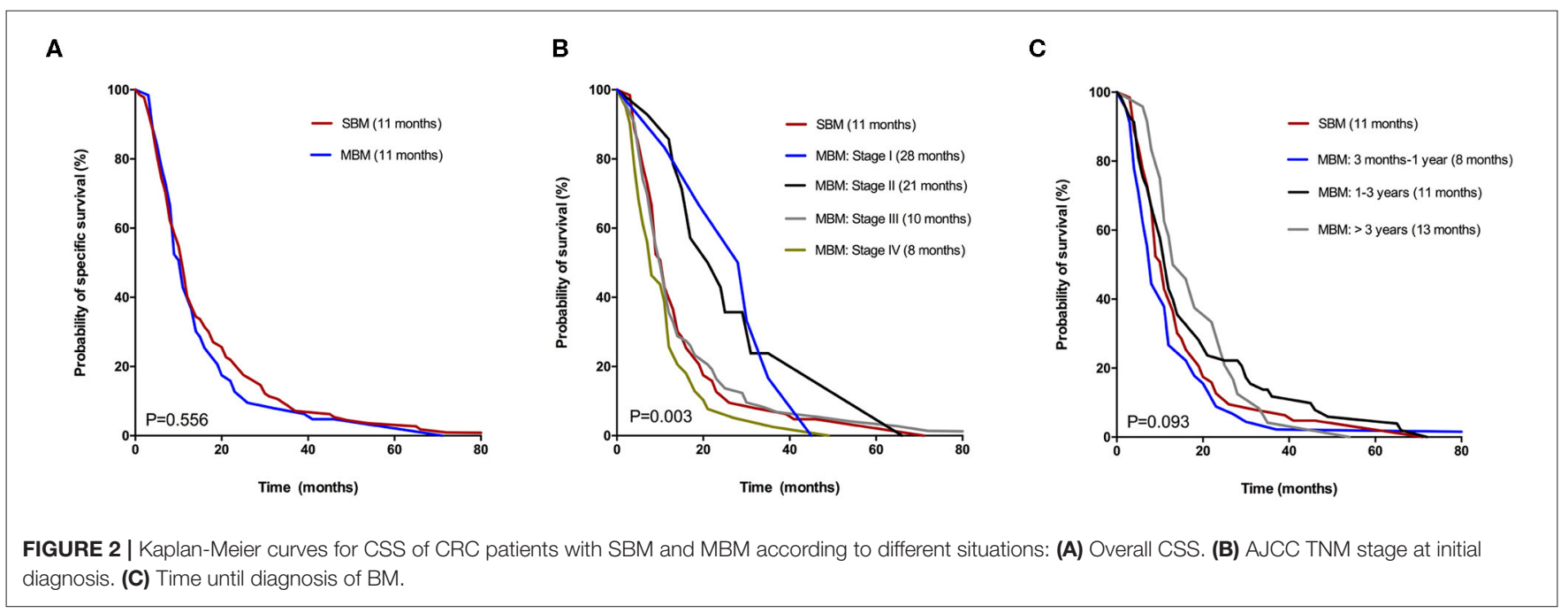


A

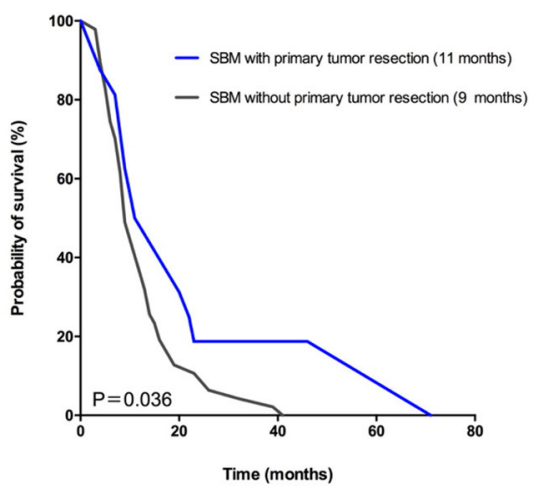

B

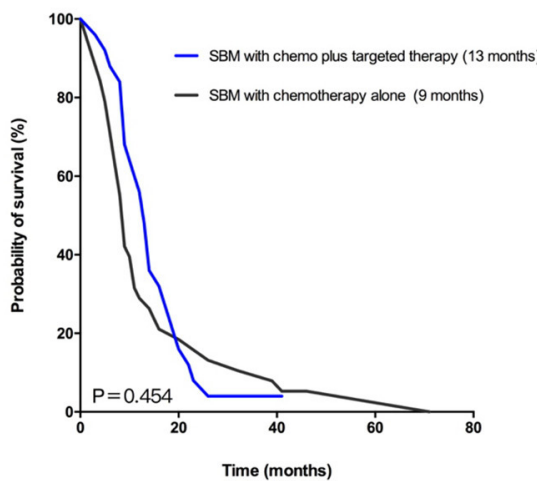

C
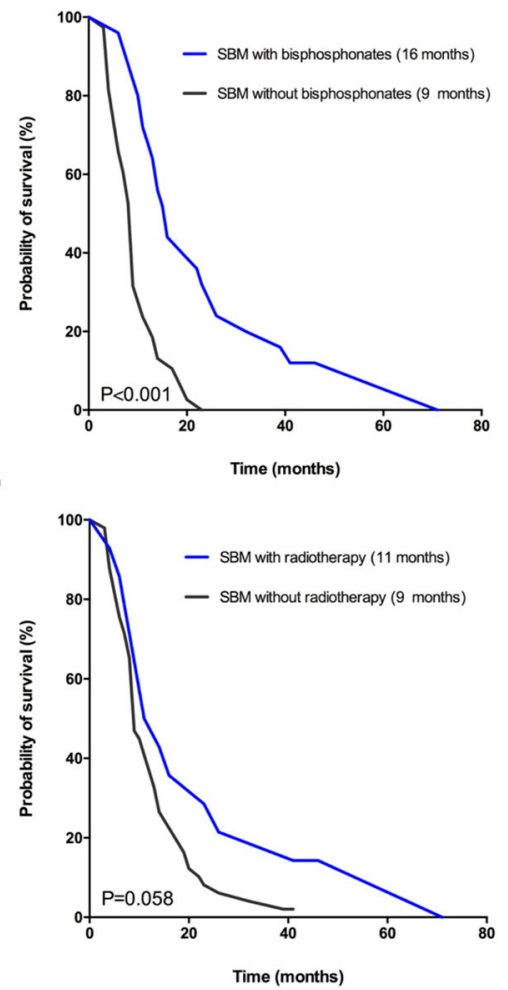
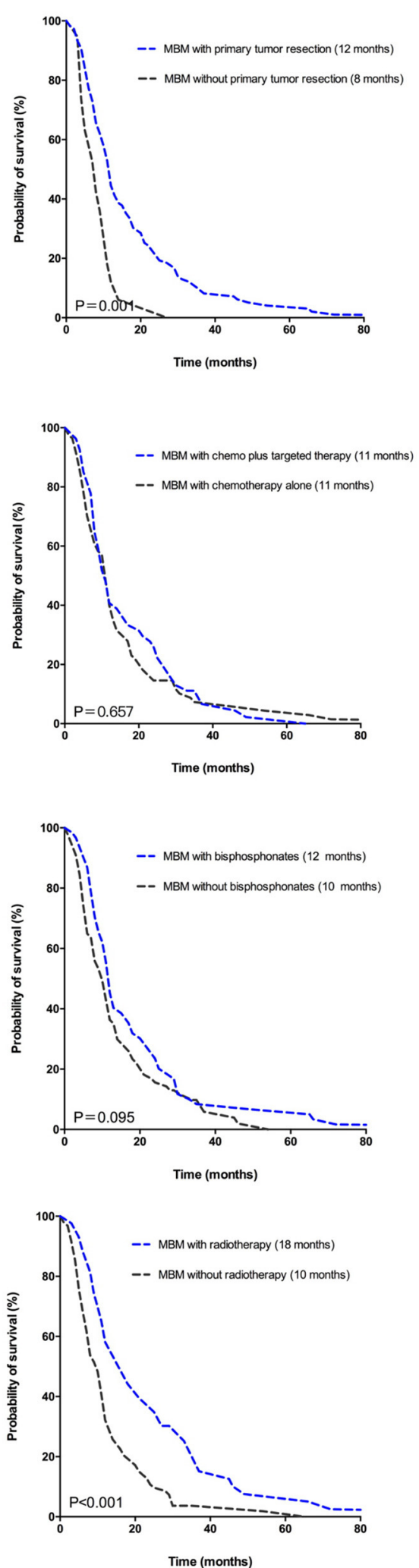

FIGURE 3 | Kaplan-Meier curves for CSS of CRC patients in synchronous group (?) versus metachronous group (-) according to various treatments: (A) Primary tumor resection. (B) Systemic treatment after BM diagnosis. (C) Bisphosphonates for BM. (D) Radiotherapy for BM. 
TABLE 3 | The univariate Kaplan-Meier analysis in CRC patients with SBM and MBM.

\begin{tabular}{|c|c|c|c|c|c|c|}
\hline \multirow{2}{*}{ Variable } & \multicolumn{3}{|c|}{ Synchronous } & \multicolumn{3}{|c|}{ Metachronous } \\
\hline & Median CSS (months) & $95 \% \mathrm{Cl}$ & $p$-value & Median CSS (months) & $95 \% \mathrm{Cl}$ & $p$-value \\
\hline Age at BM diagnosis, years & & & 0.564 & & & 0.521 \\
\hline$<60$ & 9 & $8.2-9.8$ & & 11 & $9.0-13.0$ & \\
\hline$\geq 60$ & 11 & $7.9-14.1$ & & 12 & $10.7-13.4$ & \\
\hline Gender & & & 0.617 & & & 0.705 \\
\hline Female & 10 & $7.7-12.3$ & & 11 & $8.8-13.2$ & \\
\hline Male & 11 & $8.3-13.7$ & & 11 & $9.6-12.4$ & \\
\hline Basic disease & & & 0.494 & & & 0.209 \\
\hline No & 11 & $9.2-12.8$ & & 12 & $10.5-13.5$ & \\
\hline Yes & 9 & $6.0-12.0$ & & 11 & $9.3-12.7$ & \\
\hline Primary tumor location & & & 0.990 & & & 0.273 \\
\hline Rectum & 11 & $8.0-14.0$ & & 12 & $10.3-13.7$ & \\
\hline Left hemicolon & 11 & $6.0-16.0$ & & 11 & $7.9-14.1$ & \\
\hline Right hemicolon & 9 & $9.5-12.5$ & & 9 & $6.4-11.6$ & \\
\hline Pathological type of tumor & & & 0.766 & & & 0.516 \\
\hline Adenocarcinoma & 11 & $8.6-13.4$ & & 11 & $9.8-12.2$ & \\
\hline Signet-ring cell carcinoma & 4 & $1.1-6.9$ & & 8 & $5.0-11.0$ & \\
\hline Others & 8 & $1.6-14.4$ & & NA & NA & \\
\hline Tumor grade & & & 0.494 & & & 0.539 \\
\hline Grade I, II & 13 & $9.0-17.0$ & & 12 & $10.7-13.3$ & \\
\hline Grade III, IV & 9 & $8.2-9.8$ & & 8 & $5.9-10.1$ & \\
\hline UK & 9 & $5.2-12.8$ & & 9 & $5.4-12.6$ & \\
\hline AJCC TNM stage at initial diagnosis & & & NA & & & $0.003^{\star}$ \\
\hline I & NA & NA & & 28 & $14.8-41.2$ & \\
\hline$\|$ & NA & NA & & 21 & $8.2-33.8$ & \\
\hline III & NA & NA & & 10 & $8.2-11.8$ & \\
\hline IV & 11 & $9.6-12.4$ & & 8 & $4.3-11.7$ & \\
\hline UK & NA & NA & & 11 & $0.0-29.6$ & \\
\hline CEA levels at BM diagnosis & & & 0.139 & & & $0.008^{\star}$ \\
\hline Negative & 11 & $7.1-14.9$ & & 11 & $8.9-13.1$ & \\
\hline Positive & 10 & $8.1-11.9$ & & 11 & $9.2-12.8$ & \\
\hline UK & 8 & $0.0-16.4$ & & 12 & $6.2-17.8$ & \\
\hline CA199 levels at BM diagnosis & & & 0.367 & & & $<0.001^{*}$ \\
\hline Negative & 11 & $7.6-14.4$ & & 13 & $9.0-17.0$ & \\
\hline Positive & 9 & $8.0-10.0$ & & 8 & $6.5-9.5$ & \\
\hline UK & 13 & $0.2-25.8$ & & 12 & $6.2-17.8$ & \\
\hline ALP levels at BM diagnosis & & & $0.023^{\star}$ & & & $0.024^{\star}$ \\
\hline Negative & 11 & $7.6-14.4$ & & 12 & $10.9-13.1$ & \\
\hline Positive & 8 & $5.6-10.4$ & & 8 & $2.7-13.3$ & \\
\hline UK & 4 & NA & & 10 & $4.9-15.1$ & \\
\hline Bone involvement & & & $0.001^{\star}$ & & & $0.034^{\star}$ \\
\hline Solitary & 11 & $8.9-13.1$ & & 15 & $10.4-19.6$ & \\
\hline Multiple & 5 & $3.9-6.1$ & & 10 & $8.0-12.0$ & \\
\hline KPS at BM diagnosis & & & $0.001^{*}$ & & & 0.446 \\
\hline$\geq 80$ & 11 & $8.1-13.9$ & & 12 & $10.7-13.3$ & \\
\hline$<80$ & 5 & $1.3-8.7$ & & 7 & $1.3-12.7$ & \\
\hline Extra-osseous metastases & & & 0.603 & & & 0.224 \\
\hline No & 11 & $8.7-13.3$ & & 12 & $5.4-18.6$ & \\
\hline Yes & 10 & $8.2-11.8$ & & 11 & $9.4-12.6$ & \\
\hline
\end{tabular}

(Continued) 
TABLE 3 | Continued

\begin{tabular}{|c|c|c|c|c|c|c|}
\hline Variable & \multicolumn{3}{|c|}{ Synchronous } & \multicolumn{3}{|c|}{ Metachronous } \\
\hline 3 months -1 year & & NA & & 8 & $6.7-9.3$ & \\
\hline $1-3$ years & & NA & & 12 & $10.4-13.6$ & \\
\hline$>3$ years & & NA & & 13 & $7.0-19.0$ & \\
\hline No & 9 & $7.0-11.0$ & & 8 & $1.2-10.8$ & \\
\hline Yes & 11 & $3.2-18.8$ & & 12 & $10.7-13.3$ & \\
\hline $\begin{array}{l}\text { Systemic treatment after BM } \\
\text { diagnosis }\end{array}$ & & & 0.454 & & & 0.657 \\
\hline Chemotherapy alone & 9 & $7.8-10.2$ & & 11 & $9.7-12.3$ & \\
\hline Chemotherapy plus targeted therapy & 13 & $11.0-15.0$ & & 11 & $8.8-13.2$ & \\
\hline Radiotherapy for BM & & & $0.058^{*}$ & & & $<0.001^{*}$ \\
\hline No & 9 & $7.6-10.4$ & & 10 & $8.1-11.9$ & \\
\hline Yes & 11 & $5.5-16.5$ & & 18 & $11.6-24.4$ & \\
\hline Metastasectomy for BM & & & NA & & & 0.541 \\
\hline No & NA & NA & & 11 & $9.6-12.4$ & \\
\hline Yes & NA & NA & & 21 & $5.0-37.0$ & \\
\hline
\end{tabular}

An asterisk (*) indicated variables with p-value < 0.10, which was selected into multivariable COX regression. CRC, colorectal cancer; SBM, synchronous bone metastasis; MBM, metachronous bone metastasis; Cl, confidence interval; NA, not available; CEA, carcinoembryonic antigen; CA199, carbohydrate antigen199; ALP, alkaline phosphatase.

difficult to interpret this result because the CSS of MBM patients significantly varied by different AJCC stages.

The clinical outcomes of patients with SBM and MBM appeared to be affected by different clinicopathological characteristics. We found CA199 levels was an independent prognostic factor only for MBM patients. But the CSS of patients with positive ALP levels was shorter in both two groups by univariate analysis, which was consistent with previous studies (13). So, careful surveillance in those indicators for patients with $\mathrm{BM}$ from CRC is recommended. Most researches have revealed the relationship between multiple BMs and worse prognosis $(17,18)$, while minority of studies have demonstrated the prognosis of CRC patients has no association with the number of BMs (19). In our study, multiple bone involvement was related to shorter survival. The median CSS of patients with multiple and solitary bone involvement was 5 and 11 months in SBM group $(p<0.001)$ and 10 and 15 months in MBM group $(p=$ $0.034)$, respectively. Therefore, systematic imaging examination is helpful to assess the outcome of BM.

The association between TNM stage and overall survival of CRC is generally confirmed (20). In our study, MBM patients with stage I at initial diagnosis had best prognosis with median CSS of 28 months, while it dramatically decreased to 8 months for those with stage IV ( $p=0.003)$. Thus, strengthening early diagnosis of CRC and active treatment might also prolong the CSS even the BM was developed metachronously.

The prognosis of patients with SBM and MBM was also affected by distinct provision of treatment. As the rare metastatic disease, standard treatment guidelines for CRC patients with BM have not been established. Because all cases were treated with palliative chemotherapy after BM diagnosis in our study, the utility of chemotherapy in each group was unclear.

Bisphosphonates therapy can prevent the occurrence of osteolytic lesions and SREs caused by BM, which has become an effective treatment for bone pain and hypercalcemia $(21,22)$. Commonly used bisphosphonates such as pamidronate, zoledronic acid and ibandronate can be treated for BM patients in combination with conventional anti-tumor drugs. The difference in CSS of patients with and without bisphosphonates is significant only in SBM group, implying the sensitivity to bisphosphonates for SBM and MBM patients might exist difference.

Local treatments of CRC with BM include radiotherapy and surgery, etc. Previous researches have revealed radiotherapy can reduce bone pain and prevent pathological fracture or spinal cord compression (23-25). According to our study, median CSS of patients with palliative radiotherapy was significantly prolonged only in MBM group (18 vs. 10 months, $p<0.001$ ), which was also found to be one of independent prognostic factors for MBM patients. A meta-analysis of 1,026 cases from retrospective studies had suggested an improved survival for stage IV CRC patients with primary tumor resection (26). Another recent research enrolled 3,423 patients, reporting a poor prognosis for the patients with synchronous metastases 


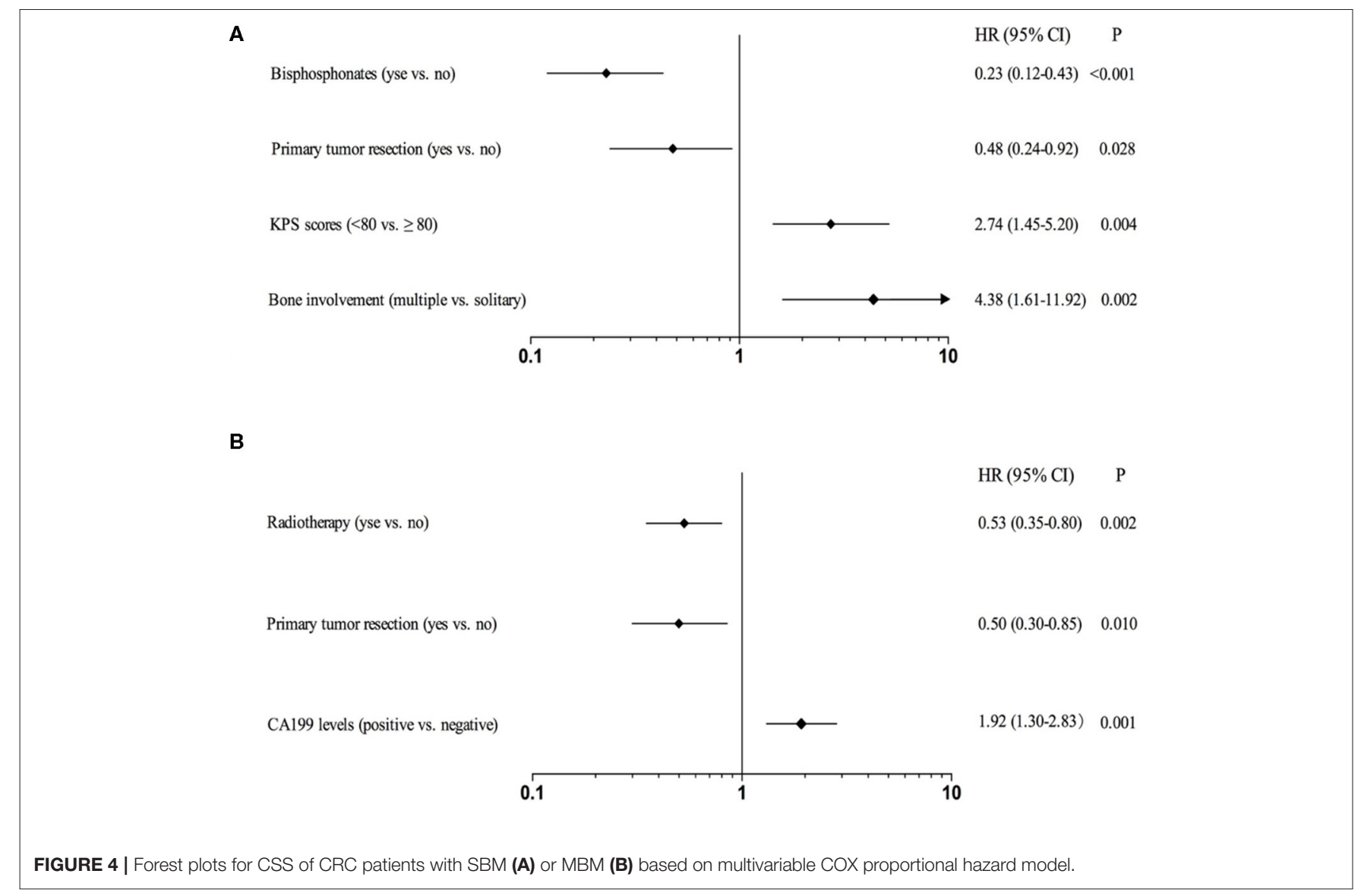

who did not receive the resection of primary tumor (27). Despite these evidences, primary tumor resection has not been confirmed as a factor related to prolonged outcome of patients with unresectable synchronous metastases (28, 29). Our study showed that patients with primary tumor resection had significantly longer CSS in SBM group (11 vs. 9 months, $p=0.036$ ), which was also an independent prognostic factor. This might be attributable to the reduction of tumorrelated complications such as systemic inflammation, bleeding, obstruction and perforation. If advanced patients can tolerate the operation, active treatment for $\mathrm{CRC}$ is an alternative method for improving the survival of BM (30). Only 3 patients underwent operative treatment of $\mathrm{BM}$ due to spinal cord compression in our study and we could not evaluate its' effect on outcome of patients. When non-operative treatment for pathological fracture, spinal instability or other complications caused by BM is invalid, surgical treatment for BM could be considered.

Our study had some limitations. First, this was a retrospective and single-center study, selection bias might occur. Second, the modest samples and non-randomized design limited the generalizability for the conclusions regarding optimal clinical management. Therefore, further prospective researches with randomized design, large sample and more clinical features are warranted.

\section{CONCLUSION}

Our study compared the clinical data and outcomes of SBM and MBM patients from CRC. Meanwhile, we identified favorable clinicopathological characteristics and treatments in SBM and MBM group, respectively. That could potentially guide physicians to treat patients with distinct clinical intervention and therapeutic strategies.

\section{DATA AVAILABILITY STATEMENT}

The datasets generated for this study are available on request to the corresponding author.

\section{ETHICS STATEMENT}

The studies involving human participants were reviewed and approved by Ethics Committee of National Cancer Center/Cancer Hospital, Chinese Academy of Medical Sciences. Written informed consent for participation was not required for this study in accordance with the national legislation and the institutional requirements. 


\section{AUTHOR CONTRIBUTIONS}

XW: writing-review and editing, supervision. XG: conceptualization, investigation, and supervision. CM: conceptualization, investigation, and writing-original draft. RW: software and formal analysis. SW: validation and data curation. JQ: resources. ZZ: validation. HC: investigation and project administration. ZL: data curation and supervision. ZJ:

\section{REFERENCES}

1. Benson AB, Venook AP, Cederquist L, Chan E, Chen YJ, Cooper HS, et al. Colon cancer, version 1.2017, NCCN clinical practice guidelines in oncology. J Natl Compr Canc Netw. (2017) 15:370-98. doi: 10.6004/jnccn. 2017.0036

2. Malvezzi M, Carioli G, Bertuccio P, Boffetta P, Levi F, La Vecchia C, et al. European cancer mortality prediction for the year 2017, with focus on lung cancer. Ann Oncol. (2017) 28:1117-23. doi: 10.1093/annonc/ $\mathrm{mdx} 033$

3. Freddie B, Jacques F, Isabelle S. Global cancer statistics 2018: GLOBOCAN estimates of incidence and mortality worldwide for 36 cancers in 185 countries. CA Cancer J Clin. (2018) 68:394-424. doi: 10.3322/caac. 21492

4. Christensen T, Jensen S, Larsen F, Nielsen D. Systematic review: incidence, risk factors, survival and treatment of bone metastases from colorectal cancer. J Bone Oncol. (2018) 13:97-105. doi: 10.1016/j.jbo.2018. 09.009

5. Sundermeyer ML, Meropol NJ, Rogatko A, Wang H, Cohen SJ. Changing patterns of bone and brain metastases in patients with colorectal cancer. Clin Colorectal Cancer. (2006) 5:108-13. doi: 10.3816/CCC.2005. n.022

6. Faisal S, Saivaishnavi K, Jordan C, Zhuo L, Dorin C, Amit $\mathrm{M}$, et al. Characteristics and outcomes of patients with colorectal cancer and bone metastasis. J Clin Oncol. (2019) 37:e15063. doi: 10.1200/JCO.2019.37.15_suppl.e15063

7. Katoh M, Unakami M, Hara M, Fukuchi S. Bone Metastasis for colorectal cancer in autopsy cases. J Gastroenterol. (1995) 30:61518. doi: 10.1007/BF02367787

8. Khattak MA, Martin HL, Beeke C, Price T, Carruthers S, Kim S, et al. Survival differences in patients with metastatic colorectal cancer and with single site metastatic disease at initial presentation: results from South Australian clinical registry for advanced colorectal cancer. Clin Colorectal Cancer. (2012) 11:247-54. doi: 10.1016/j.clcc.2012. 06.004

9. Santini D, Tampellini M, Vincenzi B, Ibrahim T, Ortega C, Virzi V, et al. Natural history of bone metastasis in colorectal cancer: final results of a large Italian bone metastases study. Ann Oncol. (2012) 23:207277. doi: 10.1093/annonc/mdr572

10. Van der Geest L, Lanm-Boer J, Koopman M, Verhoef C, Elferink M, de Wilt J. Nationwide trends in incidence, treatment and survival of colorectal cancer patients with synchronous metastases. Clin Exp Metastasis. (2015) 32:457-65. doi: 10.1007/s10585-015-9719-0

11. Bockhorn M, Frilling A, Frühauf NR, Neuhaus J, Molmenti E, Trarbach T, et al. Survival of patients with synchronous and metachronous colorectal liver metastases - is there a difference? J Gastrointest Surg. (2008) 12:1399405. doi: 10.1007/s11605-008-0508-9

12. Ghiringhelli F, Hennequin A, Drouillard A, Lepage CM, Faivre J, Bouvier AM. Epidemiology and prognosis of synchronous and metachronous colon cancer metastases: A French population-based study. Dig Liver Dis. (2014) 46:854-58. doi: 10.1016/j.dld.2014.05.011

13. Mekenkamp L, Koopman M, Teerenstra S, van Krieken J, Mol L, Nagtegaal I, et al. Clinicopathological features and outcome in advanced colorectal cancer patients with synchronous vs metachronous metastases. Br J Cancer. (2010) 103:159-64. doi: 10.1038/sj.bjc.6605737 data curation and revision. All authors contributed to the article and approved the submitted version.

\section{FUNDING}

This work was supported by CAMS Innovation Fund for Medical Sciences (CIFMS) (2016-I2M-1-001) and Beijing Science and Technology Program (D17110002617004).

14. Colloca G, Venturino A, Guarneri D. Different variables predict the outcome of patients with synchronous versus metachronous metastases of colorectal cancer. Clin Transl Oncol. (2020). doi: 10.1007/s12094-01902277-7. [Epub ahead of print].

15. Donskov F, Xie W, Overby A, Wells J, Fraccon A, Sacco C, et al. Synchronous versus metachronous metastatic disease: impact of time to metastasis on patient outcome-results from the international metastatic renal cell carcinoma database consortium. Eur Urol Oncol. (2020). doi: 10.1016/j.euo.2020.01.001. [Epub ahead of print].

16. Flannery T, Suntharalingam M, Kwok Y, Koffman B, Amin P, Chin L, et al. Gamma knife stereotactic radiosurgery for synchronous versus metachronous solitary brain metastases from non-small cell lung cancer. Lung Cancer. (2003) 42:327-33. doi: 10.1016/S0169-5002(03)00357-X

17. Park HS, Rha SY, Kim HS, Hyung WJ, Park JS, Chung HC, et al. A prognostic model to predict clinical outcome in gastric cancer patients with bone metastasis. Oncology. (2011) 80:142-50. doi: 10.1159/000 328507

18. Choi D, Fox Z, Albert T, Arts M. Prediction of quality of life and survival after surgery for symptomatic spinal metastases: a multicenter cohort study to determine suitability for surgical treatment. Neurosurgery. (2015) 77:698708. doi: 10.1227/NEU.0000000000000907

19. Wibmer C, Leithner A, Hofmann G, Clar H, Windhager R. Survival analysis of 254 patients after manifestation of spinal metastases evaluation of seven preoperative scoring systems. Spine. (2011) 36:1977-86. doi: 10.1097/BRS.0b013e3182011f84

20. O'Connell JB, Maggard MA, Ko CY. Colon cancer survival rates with the new american joint committee on cancer sixth edition staging. J Natl Cancer. (2004) 96:1420-5. doi: 10.1093/jnci/djh275

21. Liu J, Huang W, Zhou R, Jia S, Tang W, Luo Y, et al. Bisphosphonates in the treatment of patients with metastatic breast, lung, and prostate cancer. Medicine. (2015) 94:e2014. doi: 10.1097/MD.0000000000 002014

22. Roemer-Becuwe C, Krakowski I, Conroy T. Bisphosphonates, pain and quality of life in metastatic breast cancer patients: a literature review. Bull Cancer. (2003) 90:1097-105

23. Mcdonald R, Ding K, Brundage M, Meyer RM, Chow E. Effect of radiotherapy on painful bone metastases: a secondary analysis of the NCIC clinical trials group symptom control trial SC.23. JAMA Oncol. (2017) 3:9539. doi: 10.1001/jamaoncol.2016.6770

24. Edward C, Kristin H, Grace F, May T, Sze WM. Palliative radiotherapy trials for bone metastases: a systematic review. J Clin Oncol. (2007) 25:142336. doi: 10.1200/JCO.2006.09.5281

25. Wang X, Rhines L, Shiu A, Yang J, Selek U, Gning I, et al. Stereotactic body radiation therapy for management of spinal metastases in patients without spinal cord compression: a phase 102 trial. Lancet Oncol. (2012) 13:395-402. doi: 10.1016/S1470-2045(11)70384-9

26. Stillwell AP, Buettner P, Ho Y. Meta-analysis of survival of patients with stage IV colorectal cancer managed with surgical resection versus chemotherapy alone. World J Surg. (2010) 34:797-807. doi: 10.1007/s00268-0090366-y

27. Van Rooijen KL, Shi Q, Goey KKH, Meyers J, Heinemann V, Diaz-Rubio $\mathrm{E}$, et al. Prognostic value of primary tumour resection in synchronous metastatic colorectal cancer: individual patient data analysis of first-line randomised trials from the ARCAD database. Eur J Cancer. (2018) 91:99106. doi: 10.1016/j.ejca.2017.12.014 
28. Alawadi Z, Phatak UR, Hu CY, Bailey CE, Chang GJ. Comparative effectiveness of primary tumor resection in patients with stage IV colon cancer. Cancer. (2016) 123:1124-33. doi: 10.1002/cncr.30230

29. Wong SF, Wong HL, Field K, Kosmider S, Tie J, Wong R, et al. Primary tumor resection and overall survival in patients with metastatic colorectal cancer treated with palliative intent. Clin Colorectal Cancer. (2016) 15:e12532. doi: 10.1016/j.clcc.2015.12.010

30. Turner N, tran B, Tran P, Sinnathamby M, Wong H, Jones I, et al. Primary tumor resection in patients with metastatic colorectal cancer is associated with reversal of systemic inflammation and improved survival. Clin Colorectal Cancer. (2015) 14:185-91. doi: 10.1016/j.clcc.2015.02.004
Conflict of Interest: The authors declare that the research was conducted in the absence of any commercial or financial relationships that could be construed as a potential conflict of interest.

Copyright (C) 2020 Ma, Guan, Wei, Wang, Quan, Zhao, Chen, Liu, Jiang and Wang. This is an open-access article distributed under the terms of the Creative Commons Attribution License (CC BY). The use, distribution or reproduction in other forums is permitted, provided the original author(s) and the copyright owner(s) are credited and that the original publication in this journal is cited, in accordance with accepted academic practice. No use, distribution or reproduction is permitted which does not comply with these terms. 